\title{
Long Intergenic Noncoding RNA 00152 Promotes Glioma Cell Proliferation and Invasion by Interacting with MiR-16
}

\author{
Xin Chen ${ }^{a}$ Deheng Li ${ }^{a}$ Yang Gao ${ }^{a}$ Wei Tang ${ }^{a}$ Lao IW ${ }^{b}$ Yiqun Cao ${ }^{a}$ Bin Hao \\ aDepartment of Neurosurgery, Fudan University Shanghai Cancer Center, Department of Oncology, \\ Shanghai Medical College, Fudan University, Shanghai, 'Department of Pathology, Fudan University \\ Shanghai Cancer Center, Shanghai, China
}

\section{Key Words}

Linc00152 • MiR-16 • BMI1 • Glioma • Proliferation • Invasion

\begin{abstract}
Background/Aims: Long noncoding RNAs (IncRNAs) are a novel class of protein-noncoding transcripts that are aberrantly expressed in multiple diseases including cancers. LINC00152 has been identified as an oncogene involved in many kinds of cancer; however, its expression pattern and function in human glioma remain unclear. Methods: Quantitative real-time polymerase chain reaction was carried out to measure LINC00152 expression in human glioma cell lines and tissues. CCK-8 and EdU assays were performed to assess cell proliferation, and scratch assays and Transwell assays were used to assess cell migration and invasion, respectively. Luciferase reporter assays were carried out to determine the interaction between miR-16 and LINC00152. In vivo experiments were conducted to assess tumor formation. Results: LINC00152 was found to be significantly upregulated in human glioma cell lines and clinical samples. Knockdown of LINC00152 suppressed glioma cell proliferation, migration, and invasion in vitro. In vivo assays in nude mice confirmed that LINC00152 knockdown inhibits tumor growth. Furthermore, mechanistic investigation showed that LINC00152 binds to miR16 in a sequence-specific manner and suppresses its expression. miR-16 inhibition strongly attenuated LINC00152 knockdown-mediated suppressive effects on proliferation, migration, and invasion. Moreover, LINC00152 induced BMI1 expression by sponging miR-16; this effect further promoted glioma cell proliferation and invasion. Conclusion: We regard LINC00152 as an oncogenic IncRNA promoting glioma cell proliferation and invasion and as a potential target for human glioma treatment.
\end{abstract}

Xin Chen and Deheng Li contributed equally to this work.

Yiqun CaO

and Bin $\mathrm{HaO}$

Fax+86 21 64175590, E-Mail fudancaoyiqun@163.com, hao72bin@163.com 


\section{Introduction}

Gliomas are the most pervasive and aggressive major type of primary tumor in the nervous system and have fatal outcomes because of their high invasiveness. Current therapy for these primary brain cancers is inadequate, and approximately $95 \%$ of patients succumb to the disease within 5 years of diagnosis [1]. Without treatment, the average life expectancy among patients with glioblastoma is only 4.5 months. The recommendations from the European Association focus on pathological and radiological diagnostics and pharmacotherapy [2]. Nonetheless, gliomas are frequently resistant to these therapies and are rarely curable, especially malignant glioblastoma [1]. Therefore, investigating effective strategies and treatments for the early diagnosis of glioma is urgently necessary.

Long noncoding RNAs (lncRNAs), which are defined as endogenous, cellular, proteinnoncoding RNAs more than 200 nucleotides in length, have been shown to play important roles in gene expression regulation and various aspects of tumor cellular homeostasis, including tumor cell proliferation, differentiation, apoptosis, and metastasis [3]. Long intergenic noncoding RNA 152 (LINC00152), a lncRNA whose gene is located in chromosome region 2p11.2, was first identified in gastric cancer and shows significantly increased expression in cancer tissues and cells [4]. It is involved in cell proliferation, apoptosis, epithelial-mesenchymal transition, migration, and invasiveness of gastric cancer cells [5-7]. Subsequent studies showed that LINC00152 carries out oncogenic tasks in several types of cancer, such as gallbladder cancer, lung adenocarcinoma, and renal cell carcinoma [8-10]. Nevertheless, little is known about its expression and function in glioma.

In this study, we revealed that LINC00152 is highly expressed in gliomas, and its knockdown suppressed glioma cell proliferation, migration, and invasion and tumor growth. Furthermore, miR-16 was found to target LINC00152 via a conserved consensus sequence, and reciprocal inhibition between miR-16 and LINC00152 was proven. Moreover, we show that LINC00152 induces BMI1 expression by sponging miR-16, and this effect further promotes glioma cell proliferation and invasion. Taken together, our findings revealed that LINC00152 can regulate the proliferation and invasiveness of glioma cells by inhibiting the expression of miR-16.

\section{Materials and Methods}

\section{Human tissues}

Twenty samples of glioma tissues and of eight healthy brain tissues were obtained from the Fudan University Shanghai Cancer Center Tissue Bank (Shanghai, China). All the samples were snap-frozen in liquid nitrogen after surgical resection and stored at $-80^{\circ} \mathrm{C}$. The use of human tissues in this study was approved by the Clinical Research Ethics Committee of Fudan University Shanghai Cancer Center.

\section{Cell culture}

Human U87, LN229, U251, and T98G glioma cell lines were obtained from the Chinese Academy of Sciences Cell Bank (Shanghai, China) and grown in Dulbecco's modified Eagle's medium (DMEM) supplemented with $10 \%$ of fetal bovine serum (FBS). Normal human astrocytes (NHAs) were purchased from the Sciencell Research Laboratories (Carlsbad, CA) and cultured under the conditions recommended by the vendor. All the cells were maintained in a medium containing $1 \%$ of a penicillin/streptomycin solution at $37^{\circ} \mathrm{C}$ in a humidified atmosphere containing $5 \%(\mathrm{v} / \mathrm{v})$ of $\mathrm{CO}_{2}$.

Cell transfection

miR-16 mimic, miR-16 inhibitor, and their respective negative controls were purchased from RiBoBio (Guangzhou, China). The small interfering RNAs (siRNAs) targeting LINC00152 (siLINC00152_1 and siLINC00152_2) were synthesized by Genepharma (Shanghai, China) [9]. LINC00152 short hairpin RNA (shRNA) was cloned into the pLKO.1 vector, and lentiviral particle production was performed according to a previous report [11]. The coding sequence of BMI1 was amplified and cloned into pcDNA3.1 (+) to 


\section{Cellular Physiology Cell Physiol Biochem 2018;46:1055-1064

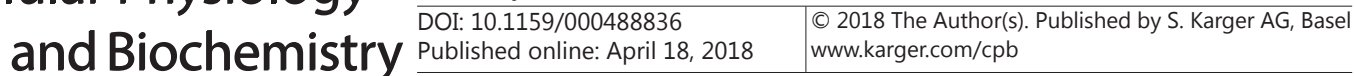 \\ Chen et al.: Roles of LINC00152 in Glioma}

generate a BMI1 expression vector. Target cells were transfected with these oligonucleotides or vectors using Lipofectamine 2000 (Life Technologies, Carlsbad, CA). The cells were harvested $48 \mathrm{~h}$ after transfection.

\section{RNA extraction and quantitative RT-PCR}

Total RNA was isolated with the TRIzol Reagent (Life Technologies). For mRNA and IncRNA quantification, RNA was reverse-transcribed into cDNA by means of random primers (Promega, Madison, WI) and M-MLV reverse transcriptase (Promega). For miR-16 quantification, total RNA was reverse-transcribed with Bulge-Loop ${ }^{\mathrm{TM}}$ microRNA-specific RT primers (RiboBio) and M-MLV reverse transcriptase (Promega). Quantitative PCRs were conducted using SYBR Premix Ex Taq II (TaKaRa, Dalian, China). GAPDH and U6 served as endogenous controls for LINC00152 and miR-16 expression, respectively. The $2^{\text {-国 } \mathrm{Ct}}$ method was applied to quantification, and fold changes in expression of target genes were normalized to the internal control. The experiments were conducted in triplicate.

\section{Cell proliferation and cell cycle analyses}

The EdU assay was performed (RiboBio), and nuclei were stained with Hoechst 33342. Images were captured by means of a fluorescence microscope (Olympus Corp., Tokyo, Japan) and analyzed in the ImageJ software (National Institutes of Health, Bethesda, MD). Cell counting Kit-8 (CCK-8, Dojin, Japan) assay was performed to assess cell proliferation. According to the vendor's protocol, transfected cells were seeded in 96-well plates at density 2000/well and cultured for 24, 48, 72, or $96 \mathrm{~h}$. Next, $10 \mu \mathrm{L}$ of the CCK-8 solution was added into each well and incubated at $37^{\circ} \mathrm{C}$ for $2 \mathrm{~h}$. The absorbance at $490 \mathrm{~nm}$ was measured. The experiments were conducted in triplicate. For cell cycle analysis, transfected cells were stained with $50 \mu \mathrm{g} /$ $\mathrm{mL}$ propidium iodide (PI) and analyzed by flow cytometry (BD Biosciences, Bedford, MD).

\section{Wound healing assay}

Transfected cells were seeded in 6-well plates and cultured until confluence reached $\sim 90 \%$. The cell layer was scratched with a tip of a $200 \mu \mathrm{L}$ pipette. After $48 \mathrm{~h}$, the wound width was measured to evaluate the wound healing ability of the tested cells.

\section{Transwell assays}

Cell migration and invasion were analyzed in Transwell chambers consisting of membrane filter inserts ( $8 \mu \mathrm{m}$ pore size; Corning, Cambridge, MA) coated or not coated with Matrigel (BD Biosciences). Transfected cells $\left(1 \times 10^{5}\right)$ were resuspended in serum-free medium and were seeded into the upper chamber. Medium with $10 \%$ of FBS was placed in the lower chamber. After incubation for $48 \mathrm{~h}$, the cells on the upper membrane surface were mechanically removed. Cells that migrated to (i.e., invaded) the lower side of the membrane were fixed with methanol and stained with a $1 \%$ crystal violet solution. The stained cells were counted under a microscope and five visual fields were imaged randomly. The results were averaged among three independent experiments.

\section{In vivo tumor growth assay}

All experimental procedures involving animals were approved by the Animal Care and Use Committee of Fudan University, China. A total of $2 \times 10^{6}$ U87 cells infected with lentiviruses expressing either shLINC00152 or shCtrl were subcutaneously injected into the flanks of BALB/c nude mice (4-6 weeks old, female, $n=6$ per group). The mice were closely monitored for tumor growth, and tumors were measured every 5 days by measuring the length and width of tumors with calipers. Tumor volumes were calculated using the equation $\mathrm{V}\left(\mathrm{mm}^{3}\right)=0.5 \times \mathrm{D} \times \mathrm{d}^{2}(\mathrm{~V}$, volume; $\mathrm{D}$, longitudinal diameter; $\mathrm{d}$, latitudinal diameter $)$. The mice were euthanized 25 days after injection, and the tumors were weighed. The proliferation index was determined by Ki-67 immunostaining and by calculating the proportion of Ki67-positive cells among all the cells in five randomly selected visual fields [12].

\section{Luciferase reporter assay}

A wild-type LINC00152 fragment containing a potential miR-16-binding site was generated and cloned into luciferase reporter vector psiCHECK-2 (Promega). The corresponding mutant construct was created by mutating the seed region of the miR-16-binding site. HEK-293T cells were seeded in 96-well plates and incubated for $24 \mathrm{~h}$, and then were cotransfected with a wild-type or mutated reporter plasmid 


\section{Cellular Physiology Cell Physiol Biochem 2018:46:1055-1064 \begin{tabular}{l|l} 
and Biochemistry Published onlıne: April18, 2018 & $\begin{array}{l}\text { (c) } 2018 \text { The Author(s). Published by S. Karger AG, Basel } \\
\text { www.karger.com/cpb }\end{array}$
\end{tabular}

and miR-16 mimic. After $48 \mathrm{~h}$ transfection, the luciferase activity was detected by means of the Dual-Luciferase Reporter Assay System (Promega). Luciferase reporter assays were performed in duplicate and repeated in three independent experiments.

\section{Statistical analysis}

SPSS 12.0 and Graph Pad Prism 5.0 software were used for statistical analysis. Data are presented as mean \pm SEM of at least three independent experiments. Student's $t$ test or one-way analysis of variance was carried out for statistical analysis when appropriate. The relation between LINC00152 and miR-16 expression was explored by Spearman's correlation analysis. Data with $\mathrm{P}<0.05$ were considered statistically significant.

\section{Results}

LINC00152 is upregulated in glioma cell lines and clinical samples

In this study, we first examined LINC00152 expression by quantitative RTPCR (qRT-PCR) in four glioma cell lines and normal human astrocytes (NHAs), and found that it was upregulated in all four glioma cell lines compared with NHAs (Fig. 1A). We also analyzed LINC00152 expression in 20 glioma tissue samples and eight healthy brain tissue samples. LINC00152 was highly expressed in glioma tissues compared with normal brain tissues (Fig. 1B). These results suggested that LINC00152 upregulation may play a biological role in glioma tumorigenesis and/or progression.

LINC00152 promotes glioma cell proliferation, migration and invasion in vitro

To assess the effect of LINC00152 on

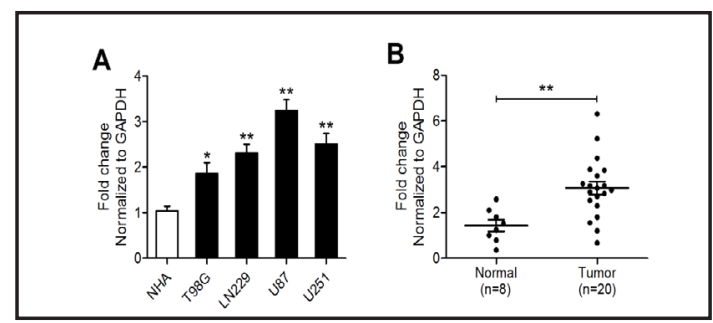

Fig. 1. LINC00152 is highly expressed in human glioma cell lines and tissues. (A) Expression levels of LINC00152 were evaluated by qRT-PCR in normal human astrocytes (NHAs) and four glioma cell lines (T98G, LN229, U87, and U251). (B) qRT-PCR analysis of LINC00152 expression in 20 glioma tissue samples and eight normal brain tissue samples. GAPDH served as the endogenous control. ${ }^{*} \mathrm{P}<0.05$, $* * \mathrm{P}<0.01$.

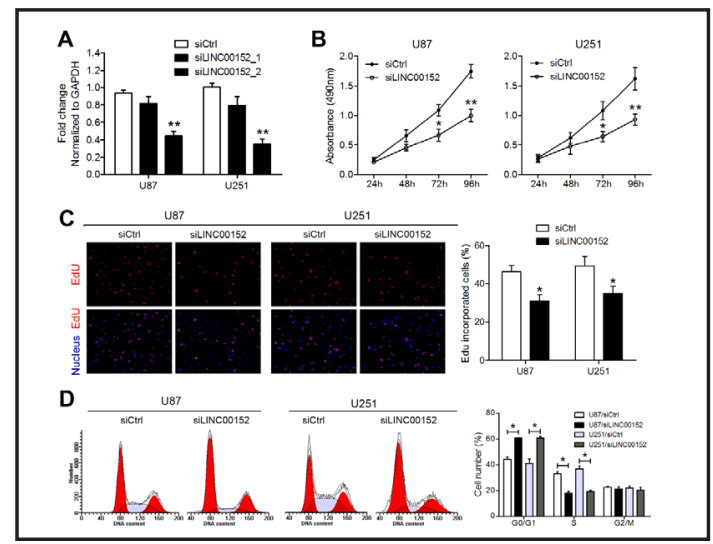

Fig. 2. Effects of LINC00152 knockdown on glioma cell proliferation in vitro. (A) Analysis of LINC00152 expression by qRT-PCR in U87 and U251 cells transfected with LINC00152-specific siRNAs (siLINC00152_1 and siLINC00152_2). (B) CCK-8 assay was performed to determine glioma cell viability following siLINC00152 silencing. (C) Representative pictures (left) and quantification (right) of EdU-positive cells. (D) Flow-cytometric assays were performed to analyze cell cycle progression. ${ }^{*} \mathrm{P}<0.05,{ }^{*} \mathrm{P}<0.01$.

the glioma cell phenotype, we synthesized two siRNAs to knock down LINC00152 expression in U87 and U251 cells, and $48 \mathrm{~h}$ after transfection, we observed that the knockdown efficiency was better for siRNA_2 (Fig. 2A). Thus, we chose this siRNA to conduct the subsequent experiments. The CCK-8 assay revealed that cell growth was suppressed when LINC00152 expression was knocked down in U87 and U251 cells compared with controls (Fig. 2B). The EdU staining assay also revealed that the knockdown of LINC00152 decreased glioma cell proliferation (Fig. 2C). Next, we determined whether this growth inhibition was associated with cell cycle changes. Flow cytometric analysis revealed that U87 and U251 cells with LINC00152 knockdown showed a marked increase in the proportion of cells in G0/G1 phases, suggesting that LINC00152 knockdown inhibited the G1-S transition of glioma 
cells (Fig. 2D). After that, to determine the effects of LINC00152 knockdown on glioma cell migration, the wound healing assay was performed. As shown in Fig. 3A, the migratory ability of U87 and U251 cells transfected with siLINC00152 was much lower than that of cells transfected with siCtrl (control). Accordingly, Transwell migration and invasion assays showed that LINC00152 knockdown inhibited the migratory ability of glioma cells by $\sim 60 \%$ and invasiveness by $\sim 50 \%$ when compared with control cells (Fig. 3B and 3C).

In contrast, LINC00152 overexpression in T98G cells, which have low endogenous LINC00152 levels, significantly increased cell proliferation, migration, and invasion (Fig. 4). These data suggested that the gene of LINC00152 may act as an oncogene and that its suppression in cancer cells may inhibit tumor progression.

\section{LINC00152 knockdown inhibits glioma cell growth in vivo}

To explore the potential functions of LINC00152 in vivo, U87 cells were infected with lentiviruses expressing shLINC00152 or shCtrl and then were injected subcutaneously into nude mice. Twenty-five days after the injection, the tumors that formed in the shLINC00152

Fig. 3. Effects of LINC00152 knockdown on glioma cell migration and invasion in vitro. (A) Representative images (upper panels) and quantification (lower panels) of the wound-healing assay of U87 and U251 cells transfected with either siLINC00152 or siCtrl. (B) Representative images (top) and quantification (bottom) of the Transwell migration assay of U87 and U251 cells transfected with either siLINC00152 or siCtrl. (C) Representative images (top) and quantification (bottom) of the Transwell invasion assay. ${ }^{*} \mathrm{P}<0.05,{ }^{* *} \mathrm{P}<0.01$.

Fig. 4. Effects of LINC00152 overexpression on glioma cell migration and invasion in vitro. (A) qRTPCR analysis of LINC00152 expression in T98G cells transfected with either pcDNA3.1-LINC00152 or an empty vector (control). LINC00152 overexpression promotes cell proliferation (B), cell cycle progression (C), and migration and invasion (D). ${ }^{*} \mathrm{P}<0.05$, ${ }^{* *} \mathrm{P}<0.01$.

Fig. 5. Inhibition of glioma cell growth in vivo via knockdown of LINC00152. U87 cells $\left(2 \times 10^{6}\right)$ infected with either the shLINC00152 lentivirus or shCtrl lentivirus were inoculated subcutaneously into nude mice $(n=6)$, and the mice were closely monitored for tumor growth. At 25 days after the inoculation, the mice were euthanized, and the tumors were excised. (A) Representative images of the tumors that formed. (B) A growth curve of tumor volumes. (C) The weight of the formed tumors. (D) qRT-PCR analysis of LINC00152 expression in the tumors. (E) Representative pictures of hematoxylin and eosin (HE) or Ki67 staining. (F) Percentages of Ki67-positive cells. ${ }^{*} \mathrm{P}<0.05,{ }^{* *} \mathrm{P}<0.01$.
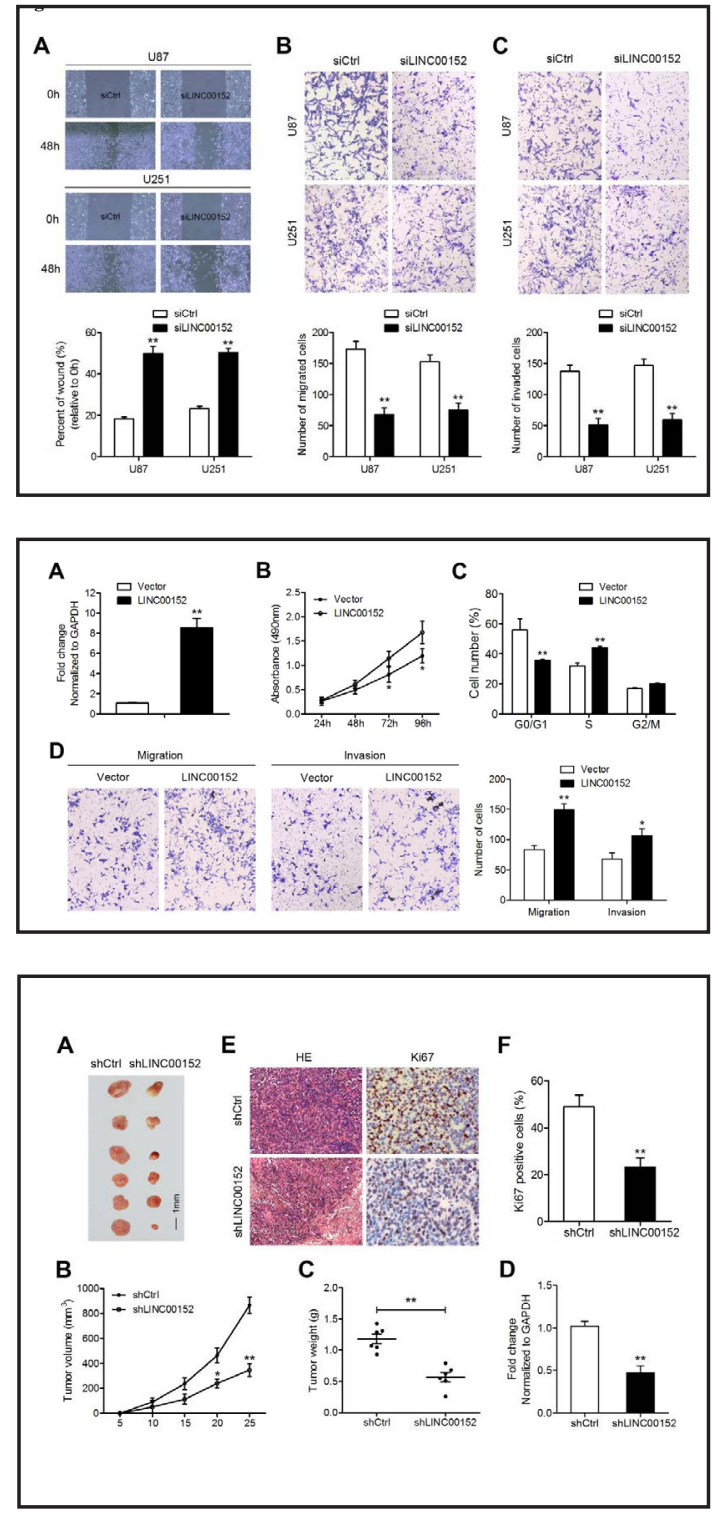


\section{Cellular Physiology Cell Physiol Biochem 2018;46:1055-1064

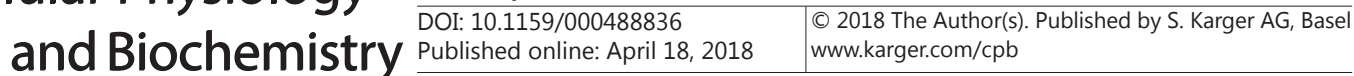 \\ Chen et al.: Roles of LINC00152 in Glioma}

group were smaller than those in the control group (Fig. 5A and 5B). In addition, the mean tumor weight was significantly lower in the shLINC00152 group than in the control group (Fig. 5C). Moreover, qRT-PCR analysis of the tumor tissues confirmed decreased LINC00152 expression in the shLINC00152 group (Fig. 5D). Immunohistochemical staining for cell proliferation marker Ki67 revealed lower percentages of Ki67-positive tumor cells in the tumors of the shLINC00152 group (Fig. 5E and 5F). These results indicated that the knockdown of LINC00152 inhibited glioma cell tumorigenesis in the nude mouse model.

\section{LINC00152 serves as a sponge for miR-16}

Given that an lncRNA has been shown to act as a competing endogenous RNA (ceRNA) or a molecular sponge for regulating the biological functions of a target microRNA (miRNA) $[13,14]$, we next aimed to determine whether LINC00152 has a similar mechanism of action in glioma. Using online miRNA target search software (http://www.mircode.org), we predicted that LINC00152 could be targeted by several miRNAs. Among them, we were particularly interested in miR-16 because of its negative effects on glioma cell proliferation and invasion $[15,16]$. The predicted binding site of miR-16 in the LINC00152 sequence is illustrated in Fig. 6A. qRT-PCR analysis showed that miR-16 expression was enhanced by LINC00152 knockdown (Fig. 6B), and that LINC00152 was downregulated by miR-16 mimic treatment (Fig. 6C). Then, a fragment of LINC00152 was inserted into a luciferase reporter system containing wild-type (Wt) or mutated (Mut) miR-16-binding site (Fig. 6A). MiR-16 was able to repress the luciferase activity of the Wt reporter vector but had no effect on the Mut vector (Fig. 6D). Furthermore, a statistically significant inverse correlation was revealed by Spearman's analysis of correlation between the levels of miR-16 and LINC00152 (Fig. 6E). These data revealed that LINC00152 is a direct target of miR-16.

To dissect the importance of miR-16 binding in LINC00152-promoted glioma progression, we transfected the miR-16 inhibitor into shLINC00152-overexpressing U87 cells (Fig. 6F). Functional assays showed that the miR-16 inhibitor largely reversed shLINC00152's inhibitory effects on glioma cell proliferation, migration, and invasion (Fig. 6G). These results strongly indicated that miR-16 plays a crucial part in LINC00152-induced promotion of glioma cells' proliferation, migration, and invasiveness.

Fig. 6. LINC00152 is a direct target of miR16 , and knockdown of miR-16 strongly attenuated LINC00152-induced inhibitory effects on glioma cell proliferation, migration, and invasion. (A) Bioinformatic prediction showed that LINC00152 sequence contains a putative binding site for miR-16. (B) miR-16 expression was increased in U87 and U251 cells transfected with siLINC00152. (C) The LINC00152 expression was decreased in U87 and U251 cells transfected with miR16 mimic. (D) A fragment of LINC00152 was cloned into a region downstream of a luciferase gene, and the whole construct was transfected into HEK-293T cells along with either the miR-16 mimic or NC

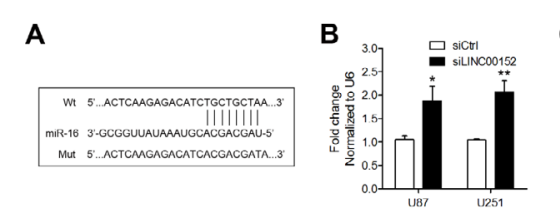

D
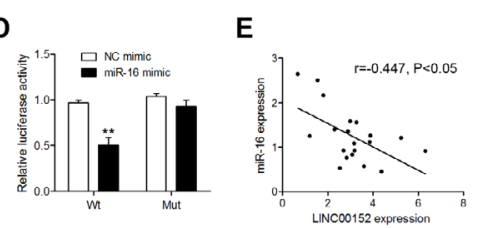

G

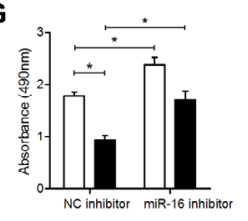

C
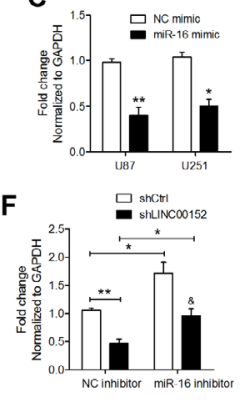

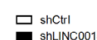
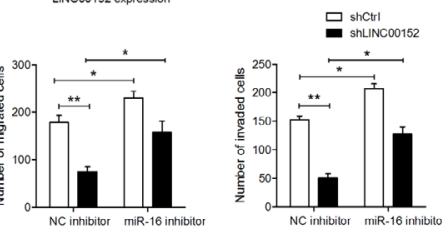

mimic. The relative luciferase activities

were inhibited in HEK293T cells transfected with the Wt reporter vector, not with the Mut reporter vector. (E) Spearman's correlation analysis revealed that miR-16 expression inversely correlated with LINC00152 levels in glioma tissues $(\mathrm{r}=-0.447$; $\mathrm{P}<0.05)$. (F) qRT-PCR analysis of LINC00152 expression in shLINC00152transfected and miR-16 inhibitor-transfected U87 cells. (G) Cell viability, migration, and invasion were determined. $* \mathrm{P}<0.05,{ }^{*} \mathrm{P}<0.01$. 


\section{Cellular Physiology Cell Physiol Biochem 2018;46:1055-1064 \begin{tabular}{ll|l} 
DOI: 10.1159/000488836 & $\begin{array}{l}\text { O 2018 The Author(s). Published by S. Karger AG, Basel } \\
\text { www.karger.com/cpb }\end{array}$ \\
\cline { 2 - 3 } and Biochemistry
\end{tabular}

BMI1 is involved in LINC00152induced promotion of glioma cell proliferation, migration, and invasion MiR-16 has been proved to participate in cell proliferation and invasion by downregulating BMI1 in many cancers, including glioma $[17,18]$. Here, we hypothesized that LINC00152 functions in glioma by enhancing BMI1 expression. To test this idea, we first measured BMI1 expression in LINC00152 knockdown cells and LINC00152-overexpressing cells. As shown in Fig. 7A, LINC00152 knockdown inhibited BMI1 expression in U87 and U251 cells, whereas its overexpression enhanced BMI1 expression in T98G cells. In agreement with other reports, miR16 mimic suppressed BMI1 expression in T98G cells (Fig. 7B). Next, T98G cells were cotransfected with the LINC00152 plasmid and BMI1 siRNA, and the protein expression of BMI1 was studied (Fig. 7C). Functional assays indicated that BMI1 suppression significantly attenuated LINC00152-promoted cell proliferation and invasiveness (Fig. 7D).

\section{Discussion}

LncRNAs have emerged as possible biological regulators of a wide range of cellular activities such as proliferation, apoptosis, migration, and invasion [19]. Over the past several years, emerging data showed that alterations in the expression of lncRNAs contribute to the pathogenesis of many human cancers, where they function as either oncogenes or tumor suppressors [20]. In the present study, we investigated participation of LINC00152 in the proliferation and invasion of glioma cells.

The aberrant expression of LINC00152 has been demonstrated to contribute to malignancy of several distinct cancer types, including gastric cancer, hepatocellular carcinoma, gallbladder cancer, lung adenocarcinoma, and renal cell carcinoma [6, 8-10, 21]. Zhao et al. reported that LINC00152 is overexpressed and involved in cell cycle arrest, apoptosis, epithelial to mesenchymal transition, cell migration and invasion in gastric cancer [6]. Ji et al. found that increased levels of LINC00152 in hepatocellular carcinoma promote cell proliferation in vitro and tumor growth in vivo by activating the mTOR signaling pathway [21]. Chen et al. recently reported that LINC00152 is upregulated in lung adenocarcinoma and is associated with a poor prognosis. Meanwhile, LINC00152's oncogenic effects are mediated in part by the epigenetic silencing of IL24 expression after binding to EZH2 [9]. In the present study, we determined the expression pattern of LINC00152 in several glioma cell lines and in the normal human astrocytes NHAs using qRT-PCR. The expression levels of LINC00152 were significantly higher in all four tested glioma cell lines than in NHAs. Furthermore, the results obtained from clinical glioma tissue samples confirmed that LINC00152 is upregulated in cancer tissues compared with healthy brain tissues, thus pointing to its possible involvement in the oncogenic transformation of the cell type in question. We subsequently confirmed that LINC00152 knockdown significantly suppresses glioma cell proliferation, migration, invasion, and tumor growth. Our results on the oncogenic role of LINC00152 are similar to the findings in a recently published paper by Yu and colleagues, where LINC00152 facilitated 


\section{Cellular Physiology Cell Physiol Biochem 2018;46:1055-1064

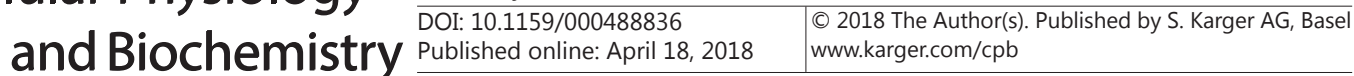 \\ Chen et al.: Roles of LINC00152 in Glioma}

cell proliferation, migration, and invasion, while suppressing apoptosis in glioma stem cells by regulating the miR-103a-3p-FEZF1-CDC25A pathway [22].

Accumulating evidence suggests that IncRNAs act as a ceRNA or miRNA sponge by binding to miRNAs and suppressing their biological functions. LINC00152 has been reported to promote renal cell carcinoma progression by epigenetically repressing P16 expression and interacting with miR-205 [10]. Additionally, LINC00152 targets miR-193a-3p-ERBB4AKT signaling and functions as a ceRNA, thereby conferring oxaliplatin resistance [23]. In the present study, using the online miRNA target search software (http://www.mircode.org), we predicted that LINC00152 may be targeted by several miRNAs, and miR-16 drew our attention. qRT-PCR analysis suggests that there is reciprocal inhibition between miR-16 and LINC00152. Furthermore, the luciferase reporter assay confirmed that miR-16 can bind to LINC00152 in a sequence-specific manner. A statistically significant inverse correlation was revealed by Spearman's analysis of correlation between the levels of miR-16 and LINC00152 in glioma tissues, suggesting that LINC00152 is a direct target of miR-16.

MiR-16, as a miRNA commonly implicated in cancer progression, has been well characterized as a potential tumor suppressor. It is frequently downregulated and plays a crucial role in oncogenesis, including the association with lower proliferation, invasion, and metastatic potential [24]. In glioma, the expression of miR-16 is low and negatively correlates with malignancy; meanwhile, restoration of miR-16 activity can significantly inhibit glioma cell growth and invasion [15]. Furthermore, miR-16 may decrease temozolomide resistance in human glioma cells [25]. In this study, we confirmed the negative effects of miR-16 on glioma cell proliferation, migration, and invasion using miR-16 inhibitor. Moreover, miR-16 inhibition strongly attenuated LINC00152 knockdown-mediated suppression of proliferation, migration, and invasion of glioma cells. These results establish a direct connection between LINC00152 and miR-16 and confirm LINC00152 as an oncogenic lncRNA in glioma cells because of the interaction with miR-16.

BMI1 is a polycomb group epigenetic gene silencer that is upregulated and involved in the development and progression of cancers [26]. It was reported that in glioma, BMI1 expression correlates with a poor prognosis and cancer progression among the patients [27] and may be regulated by several miRNAs, such as miR-218 and miR-16 [18, 28]. Here, we found that LINC00152 increases BMI1 expression indirectly by sponging miR-16. BMI1 suppression significantly reduced LINC00152-induced cell proliferation and invasion promotion, suggesting that LINC00152 functions in glioma by enhancing BMI1 expression.

In summary, we demonstrated that LINC00152 is highly expressed in gliomas, and its knockdown suppresses glioma cell proliferation, migration, invasion, and tumor growth. Furthermore, LINC00152 acts as a molecular sponge for miR-16, and there is reciprocal inhibition between miR-16 and LINC00152. MiR-16 inhibition strongly attenuated LINC00152 knockdown-mediated suppression of proliferation, migration, and invasiveness of glioma cells (Fig. 6G). Moreover, LINC00152 induces BMI1 expression by sponging miR16 , and this effect further promotes glioma cell proliferation and invasion. Thus, our findings highlight the significance of the interaction between miRNAs and lncRNAs in tumorigenesis because LINC00152 enhanced malignancy of glioma mainly by suppressing miR-16.

\section{Acknowledgements}

This work was supported by the grants from the Science and Technology Commission of Shanghai Municipality (6ZR1406300), Natural Science Foundation of China (81572921) and $\mathrm{Wu}$ Jieping Medical Foundation, China (320.6750.17150).

\section{Disclosure Statement}

No conflict of interests exists.

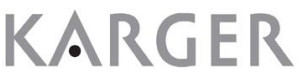




\section{Cellular Physiology Cell Physiol Biochem 2018;46:1055-1064 \begin{tabular}{l|l} 
DOI: 10.1159/000488836 & $\begin{array}{l}\text { O 2018 The Author(s). Published by S. Karger AG, Basel } \\
\text { www.karger.com/cpb }\end{array}$ \\
\hline
\end{tabular}}

\section{References}

1 Cuddapah VA, Robel S, Watkins S, Sontheimer H: A neurocentric perspective on glioma invasion. Nature Reviews Neuroscience 2014;15:455.

-2 Weller M, Van dBM, Tonn JC, Stupp R, Preusser M, Cohenjonathanmoyal E, Henriksson R, Rhun EL, Balana C, Chinot O: European association for neuro-oncology (eano) guideline on the diagnosis and treatment of adult astrocytic and oligodendroglial gliomas. Lancet Oncology 2017; 18:e315-e329.

-3 Cloutier SC, Wang S, Ma WK, Al Husini N, Dhoondia Z, Ansari A, Pascuzzi PE, Tran EJ: Regulated formation of lncrna-DNA hybrids enables faster transcriptional induction and environmental adaptation. Molecular cell 2016;62:148.

-4 Pang Q, Ge J, Shao Y, Sun W, Song H, Xia T, Xiao B, Guo J: Increased expression of long intergenic non-coding rna linc00152 in gastric cancer and its clinical significance. Tumour Biol 2014;35:5441-5447.

-5 Chen WM, Huang MD, Sun DP, Kong R, Xu TP, Xia R, Zhang EB, Shu YQ: Long intergenic non-coding rna 00152 promotes tumor cell cycle progression by binding to ezh2 and repressing p15 and p21 in gastric cancer. Oncotarget 2016;7:9773-9787.

6 Zhao J, Liu Y, Zhang W, Zhou Z, Wu J, Cui P, Zhang Y, Huang G: Long non-coding rna linc00152 is involved in cell cycle arrest, apoptosis, epithelial to mesenchymal transition, cell migration and invasion in gastric cancer. Cell Cycle 2015;14:3112-3123.

7 Zhou J, Zhi X, Wang L, Wang W, Li Z, Tang J, Wang J, Zhang Q, Xu Z: Linc00152 promotes proliferation in gastric cancer through the egfr-dependent pathway. J Exp Clin Cancer Res 2015;34:135.

-8 Cai Q, Wang ZQ, Wang SH, Li C, Zhu ZG, Quan ZW, Zhang WJ: Upregulation of long non-coding rna linc00152 by sp1 contributes to gallbladder cancer cell growth and tumor metastasis via pi3k/akt pathway. Am J Transl Res 2016;8:4068-4081.

-9 Chen QN, Chen X, Chen ZY, Nie FQ, Wei CC, Ma HW, Wan L, Yan S, Ren SN, Wang ZX: Long intergenic noncoding rna 00152 promotes lung adenocarcinoma proliferation via interacting with ezh2 and repressing il24 expression. Mol Cancer 2017;16:17.

10 Wang Y, Liu J, Bai H, Dang Y, Lv P, Wu S: Long intergenic non-coding rna 00152 promotes renal cell carcinoma progression by epigenetically suppressing p16 and negatively regulates mir-205. Am J Cancer Res 2017;7:312-322.

11 Hou S, Li N, Zhang Q, Li H, Wei X, Hao T, Li Y, Azam S, Liu C, Cheng W, Jin B, Liu Q Li M, Lei H: Xab2 functions in mitotic cell cycle progression via transcriptional regulation of cenpe. Cell Death Dis 2016;7:e2409.

12 Xia J, Wu Z, Yu C, He W, Zheng H, He Y, Jian W, Chen L, Zhang L, Li W: Mir-124 inhibits cell proliferation in gastric cancer through down-regulation of sphk1. J Pathol 2012;227:470-480.

13 Liu X-h, Sun M, Nie F-q, Ge Y-b, Zhang E-b, Yin D-d, Kong R, Xia R, Lu K-h, Li J-h: Lnc rna hotair functions as a competing endogenous rna to regulate her2 expression by sponging mir-331-3p in gastric cancer. Molecular cancer 2014;13:92.

-14 Cesana M, Cacchiarelli D, Legnini I, Santini T, Sthandier O, Chinappi M, Tramontano A, Bozzoni I: A long noncoding rna controls muscle differentiation by functioning as a competing endogenous rna. Cell 2011;147:358-369.

15 Yang TQ, Lu XJ, Wu TF, Ding DD, Zhao ZH, Chen GL, Xie XS, Li B, Wei YX, Guo LC, Zhang Y, Huang YL, Zhou YX, Du ZW: Microrna-16 inhibits glioma cell growth and invasion through suppression of bcl2 and the nuclear factor-kappab1/mmp9 signaling pathway. Cancer Sci 2014;105:265-271.

-16 Wang Q, Li X, Zhu Y, Yang P: Microrna-16 suppresses epithelial-mesenchymal transitionrelated gene expression in human glioma. Mol Med Rep 2014;10:3310-3314.

-17 Teshima K, Nara M, Watanabe A, Ito M, Ikeda S, Hatano Y, Oshima K, Seto M, Sawada K, Tagawa H: Dysregulation of bmi1 and microrna-16 collaborate to enhance an anti-apoptotic potential in the side population of refractory mantle cell lymphoma. Oncogene 2014;33:2191-2203.

18 Chen F, Chen L, He H, Huang W, Zhang R, Li P, Meng Y, Jiang X: Up-regulation of microrna-16 in glioblastoma inhibits the function of endothelial cells and tumor angiogenesis by targeting bmi-1. Anticancer Agents Med Chem 2016;16:609-620.

19 Yu Y, Yang J, Li Q, Xu B, Lian Y, Miao L: Linc00152: A pivotal oncogenic long non-coding rna in human cancers. Cell Prolif 2017;50:e12349.

20 Wang P, Liu YH, Yao YL, Li Z, Li ZQ Ma J, Xue YX: Long non-coding rna casc2 suppresses malignancy in human gliomas by mir-21. Cell Signal 2015;27:275-282. 


\section{Cellular Physiology Cell Physiol Biochem 2018;46:1055-1064

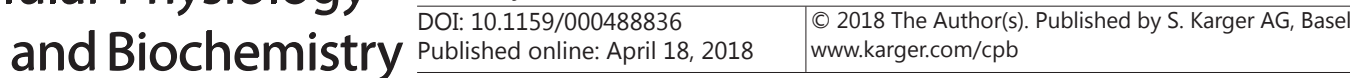 \\ Chen et al.: Roles of LINC00152 in Glioma}

-21 Ji J, Tang J, Deng L, Xie Y, Jiang R, Li G, Sun B: Linc00152 promotes proliferation in hepatocellular carcinoma by targeting epcam via the mtor signaling pathway. Oncotarget 2015;6:42813-42824.

22 Yu M, Xue Y, Zheng J, Liu X, Yu H, Liu L, Li Z, Liu Y: Linc00152 promotes malignant progression of glioma stem cells by regulating mir-103a-3p/fezf1/cdc25a pathway. Mol Cancer 2017;16:110.

23 Yue B, Cai D, Liu C, Fang C, Yan D: Linc00152 functions as a competing endogenous rna to confer oxaliplatin resistance and holds prognostic values in colon cancer. Mol Ther 2016;24:2064-2077.

-24 Huang E, Liu R, Chu Y: Mirna-15a/16: As tumor suppressors and more. Future Oncol 2015;11:2351-2363.

25 Han J, Chen Q: Mir-16 modulate temozolomide resistance by regulating bcl-2 in human glioma cells. Int J Clin Exp Pathol 2015;8:12698-12707.

-26 Glinsky GV, Berezovska 0, Glinskii AB: Microarray analysis identifies a death-from-cancer signature predicting therapy failure in patients with multiple types of cancer. J Clin Invest 2005;115:1503-1521.

27 Li J, Gong LY, Song LB, Jiang LL, Liu LP, Wu J, Yuan J, Cai JC, He M, Wang L, Zeng M, Cheng SY, Li M: Oncoprotein bmi-1 renders apoptotic resistance to glioma cells through activation of the ikk-nuclear factorkappab pathway. Am J Pathol 2010;176:699-709.

28 Tu Y, Gao X, Li G, Fu H, Cui D, Liu H, Jin W, Zhang Y: Microrna-218 inhibits glioma invasion, migration, proliferation, and cancer stem-like cell self-renewal by targeting the polycomb group gene bmi1. Cancer Res 2013;73:6046-6055. 